\title{
Convergence of a Class of Deficient Discrete Cubic Splines
}

\author{
S. S. Rana \\ Professor \\ Deptt. Of Mathematics \\ R. D. University \\ Jabalpur, India
}

\author{
Mridula Dube \\ Professor \\ Deptt. Of Mathematics \\ R. D. University \\ Jabalpur, India
}

\author{
Preeti Tiwari \\ Astt. Professor \\ Deptt. Of Mathematics \\ Gyan . Ganga Instt. Of Tech \\ and Sciences \\ Jabalpur, India
}

\begin{abstract}
In the present paper, the existence and uniqueness of deficient discrete cubic spline interpolant by matching the given function and first order difference at the intermediate points between successive mesh points for a uniform mesh has been discussed and also a error estimate concerning this deficient discrete cubic spline interpolant is obtained
\end{abstract}

\section{Keywords}

Discrete cubic spline, Difference Operators, Convergence, Error bounds, Continuity.

\section{INTRODUCTION}

Discrete splines are the splines in which smoothness of the function at mesh points is expressed in terms of differences rather than continuity of derivatives. These splines have been introduced by Mangasarian and Schumaker [10] to find solution of some minimization problems involving differences. Existence, uniqueness and convergence properties of discrete cubic spline interpolant matching the given function at mesh points and intermediate points have been studied by a number of authors (see Lyche ( [7], [8] ), Dikshit and Powar [ 3 ], Dikshit and Rana [ 4 ], and Rana ( [ 11 ], [ 12 ] ). Local behaviour of the first difference of a discrete cubic spline interpolant has been obtained by Rana and Dubey [ 12 ]. To smooth histograms, estimates of derivatives of error functions are sometimes used (see Boneva,Kendall and Stefanov [ 2 ], A unified treatment of asymptotic error expansion for both, even and odd degree interpolatory splines given by Dikshit, Sharma and Tzimbalario [5] ). To compute nonlinear splines iteratively, Malcom has used discrete splines [9]. In the direction of some constructive aspects of discrete splines, Astor and Duris [1] , Jia [6] and Schumaker [13] are being refered. In order to define discrete cubic splines, by using the following difference operators introduced in Lyche [7 ].

$$
\begin{gathered}
D_{h}{ }^{(0)} f(x)=f(x) \\
D_{h}^{(1)} f(x)=[f(x+h)-f(x-h)] / 2 h, \\
D_{h}^{(2)} f(x)=[f(x+h)-2 f(x)+f(x-h)] / h^{2} .
\end{gathered}
$$

Cconsider a mesh $\mathrm{P}$ on the interval $[0,1]$ defined by $\mathrm{P}$ : $0:=x_{0}<x_{1}<\ldots<x_{n}=1$ such that $x_{i}-x_{i-1}=p$, for $i=1,2, \ldots, n$. For a given $h>0$, suppose a real continuous function $\mathrm{s}(\mathrm{x}, \mathrm{h})$ is defined over $[0,1]$ and its restriction to $\left[x_{i-1}, x_{i}\right]$ is a polynomial $s_{i}$ of degree three or less, for $i=1,2, \ldots, n$. Then $\mathrm{s}(\mathrm{x}, \mathrm{h})$ defines a discrete cubic spline if

$$
\begin{gathered}
D_{h}^{(j)} s_{i}\left(x_{i}, h\right)=D_{h}^{(j)} s_{i+1}\left(x_{i}, h\right) \quad j=0,1 \\
\text { and } \quad i=1, \ldots, n-1 .
\end{gathered}
$$

The present paper is organized as follows: Section 2 is about existence and uniqueness of discrete cubic spline interpolant Section 3 deals with the discrete error bounds. Section 4 concludes the paper.

\section{EXISTENCE AND UNIQUENESS}

The class of all deficient discrete cubic splines is denoted by $S(3,1, P, h)$ where $S_{1}(3,1, P, h)$ denotes the class of all deficient discrete cubic splines which satisfy the periodicity condition

$D_{h}^{(j)} s\left(x_{0}, h\right)=D_{h}^{(j)} s\left(x_{n}, h\right), \quad j=0,1,2$

Considering the following interpolatory conditions for a given function $\mathrm{f}$,

$$
\begin{gathered}
s\left(\alpha_{i}, h\right)=f\left(\alpha_{i}\right) ; \quad \alpha_{i}=x_{i-1}+\alpha p \\
i=1,2, \ldots, n-1 . \\
D_{h}^{(1)} s\left(\beta_{i}, h\right)=D_{h}^{(1)} f\left(\beta_{i}\right) ; \quad \beta_{i}=x_{i-1}+\beta p \\
i=1,2, \ldots, n-1 .
\end{gathered}
$$

where $0<\alpha<1, \quad 0 \leq \beta \leq 1, \quad$ to prove the following theorem,

Theorem 2.1: Let $\mathrm{f}$ be $1-$ periodic then for any $h>0$, there exists a unique $1-$ periodic deficient discrete cubic spline $\mathrm{s}$ in the class $S_{1}(3,1, P, h)$ which satisfies interpolatory conditions (2.2) and (2.3),

if $(i) \beta=1 / 3$ and $\alpha \in(0,0.84]$

or if (ii) $\beta=2 / 3$ and $\alpha \in[0.16,1)$, 
or if (iii) $\beta=1 / 2$ and $\alpha \in(0,1)$.

Proof: Suppose in the interval $\left[x_{i-1}, x_{i}\right]$ for all i,

$$
\begin{gathered}
s_{i}(x)=s(x, h)=A_{i}+B_{i}\left(x_{i}-x\right)+C_{i}\left(x_{i}-x\right)^{2}\left(x-x_{i-1}\right) \\
+D_{i}\left(x_{i}-x\right)\left(x-x_{i-1}\right)^{2}
\end{gathered}
$$

Writing $\alpha^{*}$ for $1-\alpha, \beta^{*}$ for $1-\beta$ and setting $D_{h}^{(1)} s\left(x_{i}, h\right)=m_{i}$ for all $\mathrm{i}$, we determine $\mathrm{A}_{\mathrm{i}}, \mathrm{B}_{\mathrm{i}}, \mathrm{C}_{\mathrm{i}}$ and $\mathrm{D}_{\mathrm{i}}$ using (1.2.2) and (1.2.3) as following

$$
f\left(\alpha_{i}\right)=A_{i}+B_{i} \alpha^{*} p+C_{i} \alpha \alpha^{* 2} p^{3}+D_{i} \alpha^{*} \alpha^{2} p^{3}
$$

Taking the first difference of $(2.4)$, we get

$$
\begin{aligned}
& D_{h}^{(1)} s(x, h)=\frac{B_{i}}{2 h} {\left[\left(x_{i}-x-h\right)-\left(x_{i}-x+h\right)\right] } \\
&+\frac{C_{i}}{2 h}\left[\left(x_{i}-x-h\right)^{2}\left(x+h-x_{i-1}\right)\right. \\
&\left.-\left(x_{i}-x+h\right)^{2}\left(x-h-x_{i-1}\right)\right] \\
&+\frac{D_{i}}{2 h}\left[\left(x_{i}-x-h\right)\left(x+h-x_{i-1}\right)^{2}\right. \\
&\left.-\left(x_{i}-x+h\right)\left(x-h-x_{i-1}\right)^{2}\right]
\end{aligned}
$$

Thus taking

$$
D^{(1)} s\left(x_{i}, h\right)=m_{i}
$$

for all $i$, we have

$$
\begin{aligned}
& m_{i}=-B_{i}+h^{2} C_{i}-\left(p^{2}+h^{2}\right) D_{i} \\
& m_{i-1}=-B_{i}+\left(p^{2}+h^{2}\right) C_{i}-h^{2} D_{i}
\end{aligned}
$$

and

$$
\begin{aligned}
D_{h}^{(1)} f\left(\beta_{i}\right)= & -B_{i}+\left[p^{2} \beta^{*}\left(\beta^{*}-2 \beta\right)+h^{2}\right] C_{i} \\
& +\left[p^{2} \beta\left(2 \beta^{*}-\beta\right)-h^{2}\right] D_{i}
\end{aligned}
$$

Thus from the above relations we get

$$
A_{i}=\frac{\left[f\left(\alpha_{i}\right) p^{2} N+m_{i-1} X_{i}+m_{i} Y_{i}+D_{h}^{(1)} f\left(\beta_{i}\right) Q\right]}{p^{2} N}
$$

with

$$
\begin{aligned}
& X_{i}=\left\{\alpha^{*} p\left[h^{2} t_{1}+p^{2} t_{2}\right]+\alpha \alpha^{* 2} p^{3} t_{3}-\alpha^{*} \alpha^{2} p^{3} t_{2}\right\} \\
& Y_{i}=\left\{\alpha^{*} p\left(2 \beta h^{2}-t_{4} p^{2}\right)-\alpha \alpha^{* 2} p^{3} t_{4}+\alpha^{*} \alpha^{2} p^{3} t_{5}\right\}
\end{aligned}
$$

and

$$
\begin{aligned}
Q_{i} & =\left[\alpha^{2}(3-2 \alpha)-1\right] p^{3}-2 \alpha^{*} h^{2} p \\
N & =-6 \beta(1-\beta), t_{1}=2(1-\beta), \\
t_{2} & =\left(3 \beta^{2}-4 \beta+1\right), t_{3}=\left(1+2 \beta-3 \beta^{2}\right), \\
t_{4} & =2 \beta-3 \beta^{2}, t_{5}=3 \beta^{2}-4 \beta
\end{aligned}
$$

$$
\begin{aligned}
& B_{i}=\frac{\left.D_{h}^{(1)} f\left(\beta_{i}\right)\left(2 h^{2}+p^{2}\right)-m_{i-1}\left[h^{2} t_{1}+p^{2} t_{2}\right]+m_{i}\left[p^{2} t_{4}-2 \beta h^{2}\right)\right]}{p^{2} N} \\
& C_{i}=\frac{D_{h}^{(1)} f\left(\beta_{i}\right)-m_{i-1} t_{3}+m_{i} t_{4}}{p^{2} N} \\
& D_{i}=\frac{m_{i-1} t_{2}-m_{i} t_{5}-D_{h}^{(1)} f\left(\beta_{i}\right)}{p^{2} N}
\end{aligned}
$$

Now substituting the values of $A_{i}, B_{i}, C_{i}$ and $D_{i}$ from above relations in (2.4), we have

$$
\begin{aligned}
s(x, h)= & f\left(\alpha_{i}\right)+\frac{D_{h}^{(1)} f\left(\beta_{i}\right)}{p^{2} N}\left[Q_{i}+\left(x_{i}-x\right)\left(p^{2}+2 h^{2}\right)\right. \\
& \left.+\left(x_{i}-x\right)^{2}\left(x-x_{i-1}\right)-\left(x_{i}-x\right)\left(x-x_{i-1}\right)^{2}\right] \\
& +\frac{m_{i-1}}{p^{2} N}\left[X_{i}-\left(h^{2} t_{1}+p^{2} t_{2}\right)\left(x_{i}-x\right)\right. \\
& \left.-t_{3}\left(x_{i}-x\right)^{2}\left(x-x_{i-1}\right)+t_{2}\left(x_{i}-x\right)\left(x-x_{i-1}\right)^{2}\right] \\
& +\frac{m_{i}}{p^{2} N}\left[Y_{i}+\left(p^{2} t_{4}-2 \beta h^{2}\right)\left(x_{i}-x\right)\right. \\
& \left.+t_{4}\left(x_{i}-x\right)^{2}\left(x-x_{i-1}\right)-t_{5}\left(x_{i}-x\right)\left(x-x_{i-1}\right)^{2}\right]
\end{aligned}
$$

Now using the continuity of $\mathrm{s}(\mathrm{x}, \mathrm{h})$ at $x=x_{i}$ i.e. $s_{i}\left(x_{i}, h\right)=s_{i+1}\left(x_{i}, h\right)$, we have 


$$
\begin{aligned}
N f\left(\alpha_{i}\right) & +\frac{D_{h}{ }^{(1)} f\left(\beta_{i}\right) Q_{i}}{p^{2}}+\frac{m_{i-1} X_{i}}{p^{2}}+\frac{m_{i} Y_{i}}{p^{2}}= \\
& N f\left(\alpha_{i+1}\right)+\frac{D_{h}{ }^{(1)} f\left(\beta_{i+1}\right)\left[Q_{i}+p^{3}+2 h^{2} p\right]}{p^{2}} \\
& +\frac{m_{i}\left[X_{i+1}-\left(h^{2} t_{1}+p^{2} t_{2}\right) p\right]}{p^{2}}
\end{aligned}
$$$$
+\frac{m_{i+1}\left[Y_{i+1}+t_{4} p^{2}-2 \beta h^{2} p\right]}{p^{2}}
$$

Now collecting the coefficients of $m_{i-1}, m_{i}, m_{i+1}$,

in $(2.10)$ we have

$$
\begin{aligned}
& m_{i-1}\left[\frac{X_{i}}{p^{2}}\right]+m_{i} \frac{\left[Y_{i}-X_{i+1}+h^{2} t_{1} p+t_{2} p^{3}\right]}{p^{2}} \\
& +m_{i+1} \frac{\left[2 \beta h^{2} p-t_{4} p^{3}-Y_{i+1}\right]}{p^{2}}=F_{i}
\end{aligned}
$$

where

$$
\begin{aligned}
F_{i}=N & \left.f\left(\alpha_{i+1}\right)-f\left(\alpha_{i}\right)\right]+D_{h}^{(1)} f\left(\beta_{i+1}\right) \frac{\left[Q_{i}+p^{3}+2 h^{2} p\right]}{p^{2}} \\
& -D_{h}^{(1)} f\left(\beta_{i}\right) \frac{Q_{i}}{p^{2}}
\end{aligned}
$$

Equation (2.11) can also be written in the following simplified form

$\left[\frac{2(1-\alpha)(1-\beta) h^{2}}{p}+R_{i} p\right] m_{i-1}+\left[\frac{2 \alpha \beta h^{2}}{p}+R_{i}^{*} p\right] m_{i+1}$ $+\left[\frac{2 \beta(1-\alpha) h^{2}}{p}+\frac{2 \alpha(1-\beta) h^{2}}{p}-\left(R_{i}+R_{i}^{*}-1+6 \beta-6 \beta^{2}\right) p\right] m_{i}=F_{i}$

for $i=1,2, \ldots, n-1$.

where

$$
\begin{aligned}
& R_{i}=(1-\alpha)^{2}(1-\beta)(1+2 \alpha-3 \beta), \\
& R_{i}^{*}=\alpha^{2} \beta(3 \beta-2 \alpha),
\end{aligned}
$$

$$
\begin{aligned}
F_{i}= & -6 \beta(1-\beta)\left[f\left(\alpha_{i+1}\right)-f\left(\alpha_{i}\right)\right] \\
& +D_{h}^{(1)} f\left(\beta_{i+1}\right)\left[\alpha^{2}(3-2 \alpha) p+\frac{2 \alpha h^{2}}{p}\right] \\
& +D_{h}^{(1)} f\left(\beta_{i}\right)\left[\frac{2(1-\alpha) h^{2}}{p}\right. \\
& \left.+\left(1-\alpha^{2}(3-2 \alpha)\right) p\right]
\end{aligned}
$$

Also the above system of equations can be written in the form

$$
A M=F
$$

where $\mathrm{M}$ and $\mathrm{F}$ are the transpose of the single column vectors $\left[m_{0}, m_{1}, \ldots, m_{n}\right]$ and $\left[F_{1}, \ldots, F_{n-1}\right]$ and $\mathrm{A}$ is the coefficient matrix of order $\mathrm{n}-1$.

In order to prove Theorem 2.1, considering all the cases of Theorem 2.1 separately.

For case (i) in which $\beta=\frac{1}{3}$ and $\alpha \in(0,0.84]$, the coefficient of $m_{i+1}$ is nonnegative for $0<\alpha \leq 0.5$ and nonpositive for $0.5 \leq \alpha<0.84$. Further the coefficients of $m_{i-1}$ and $m_{i}$ are always nonnegative and nonpositive respectively. Thus, the excess of the positive value of coefficient of $m_{i}$ over the sum of the positive values of the coefficients of $m_{i-1}$ and $m_{i+1}$ is

$$
J_{1}(\alpha)=\frac{\left(1+8 \alpha-14 \alpha^{2}+4 \alpha^{3}\right)}{3}, \text { (say) }
$$

for $0<\alpha \leq 0.5$ and $\frac{1}{3}$ for $0.5 \leq \alpha<0.84$

For case (ii) in which $\beta=\frac{2}{3}$ and $\alpha \in[0.16,1)$, we observe that the coefficient of $m_{i-1}$ is nonpositive for $0.16 \leq \alpha \leq 0.5$ and nonnegative for $0.5 \leq \alpha<1$. However the coefficients of $m_{i+1}$ and $m_{i}$ are nonnegative and nonpositive respectively. Thus, the excess of the positive value of the coefficient of $m_{i}$ over the sum of the positive values of coefficients of $m_{i-1}$ and $m_{i+1}$ is $J_{2}(\alpha)=\frac{\left(-1+8 \alpha-10 \alpha^{2}+4 \alpha^{3}\right)}{3}$,(say) 
for $0.16 \leq \alpha \leq 0.5$ and $\frac{1}{3}$ for $0.5 \leq \alpha<1$.

Considering case (iii) in which $\beta=\frac{1}{2}$ and $\alpha \in(0,1)$, we observe that the coefficient of $m_{i-1}$ is nonpositive for $0<\alpha \leq 0.25$ and nonnegative for $0.25 \leq \alpha<1$. However the coefficient of $m_{i+1}$ is nonnegative for $0<\alpha \leq 0.75$ and nonpositive for $0.75 \leq \alpha<1$ and the coefficient of $m_{i}$ is always nonpositive. Thus, the excess of the positive value of the coefficient of $m_{i}$ over the sum of the positive values of coefficients of $m_{i-1}$ and $m_{i+1}$ is $J_{3}(\alpha)=\frac{\alpha\left(6-9 \alpha+4 \alpha^{2}\right)}{2}$ for $0<\alpha \leq 0.25$ and $J_{4}(\alpha)=\frac{1+\alpha}{2} \quad$ for $\quad 0.25 \leq \alpha \leq 0.75 \quad$ and $J_{5}(\alpha)=\frac{\left(1+3 \alpha^{2}-4 \alpha^{3}\right)}{2}$ for $0.75 \leq \alpha<1$.

It may be seen easily that $J_{1}(\alpha), J_{2}(\alpha), J_{3}(\alpha), J_{4}(\alpha)$ and $J_{5}(\alpha)$ are positive for corresponding values of $\alpha$. Thus, the coefficient matrix $\mathrm{A}$ of the system of equations (2.13) is invertible and its row-max norm for $\beta=\frac{1}{3}$ (or $\frac{2}{3}$ or 12$)$, that is,

$$
\mathrm{P} A^{-1} \mathrm{P} \leq K_{1}
$$

where

$K_{1}=\max _{\alpha}\left\{3, J_{1}^{-1}(\alpha), J_{2}^{-1}(\alpha), J_{3}^{-1}(\alpha), J_{4}^{-1}(\alpha), J_{5}^{-1}(\alpha)\right\}$

Theorem 2.2: Let $\mathrm{f}$ be $1-$ periodic then for any $h>0$, there exists a unique 1 -periodic deficient discrete cubic spline $\mathrm{s}$ in the class $S_{1}(3,1, P, h)$ which satisfies interpolatory conditions $(2.2)$ and $(2.3)$ if

$$
\begin{aligned}
& \text { (i) } \alpha=1 / 3 \text { and } \beta \in\left[\frac{2}{9}, 0.74\right] \quad \text { or } \text { if } \\
& \text { (ii) } \alpha=2 / 3 \text { and } \beta \in\left[0.26, \frac{7}{9}\right), \quad \text { or if } \\
& \text { (iii) } \alpha=1 / 2 \text { and } \beta \in\left[0.23, \frac{7}{9}\right] .
\end{aligned}
$$

Proof: In order to prove Theorem 2.2, considering all the cases of Theorem 2.2 separately.
For case (i) in which $\alpha=\frac{1}{3}$ and $\beta \in\left[\frac{2}{9}, 0.74\right]$, the coefficient of $m_{i-1}$ is nonnegative for $\frac{2}{9} \leq \beta \leq 0.56$ and nonpositive $0.56 \leq \beta \leq 0.74$. The coefficient of $m_{i+1}$ is always nonnegative for $\frac{2}{9} \leq \beta \leq 0.74$. Further the coefficient of $m_{i}$ is always nonpositive for $\frac{2}{9} \leq \beta \leq 0.74$

. Thus the excess of the positive value of coefficient of $m_{i}$ over the sum of the positive values of the coefficients of $m_{i-1}$ and $m_{i+1}$ is $L_{1}(\beta)=\frac{\left(-27+162 \beta-162 \beta^{2}\right)}{27}$, for $\frac{2}{9} \leq \beta \leq 0.56$ and

$$
L_{2}(\beta)=\frac{\left(13+50 \beta-90 \beta^{2}\right)}{27} \text {,for } 0.56 \leq \beta \leq 0.74
$$

For case (ii) in which $\alpha=\frac{2}{3}$ and $\beta \in\left[0.26, \frac{7}{9}\right]$, the coefficient of $m_{i-1}$ is always nonnegative for $0.26 \leq \beta \leq \frac{7}{9}$. The coefficient of $m_{i+1}$ is nonpositive for $0.26 \leq \beta \leq 0.44$ and nonnegative for $0.44 \leq \beta \leq \frac{7}{9}$. Further, the coefficient of $m_{i}$ is always nonpositive for $0.26 \leq \beta \leq \frac{7}{9}$. Thus the excess of the positive value of coefficient of $m_{i}$ over the sum of the positive values of the coefficients of $m_{i-1}$ and $m_{i+1}$ is $L_{3}(\beta)=\frac{\left(-27+130 \beta-90 \beta^{2}\right)}{27}$,

for $\quad 0.26 \leq \beta \leq 0.44$

and $\quad L_{4}(\beta)=\frac{\left(-27+162 \beta-162 \beta^{2}\right)}{27}$, for $0.44 \leq \beta \leq \frac{7}{9}$.

For case (iii) in which $\alpha=\frac{1}{2}$ and $\beta \in\left[0.23, \frac{7}{9}\right]$, the coefficient of $m_{i-1}$ is nonnegative for $0.23 \leq \beta \leq 0.67$ and nonpositive for $0.67 \leq \beta \leq \frac{7}{9}$. The coefficient of 
$m_{i+1} \quad$ is $\quad$ nonpositive for $0.23 \leq \beta \leq 0.33$ and nonnegative for $0.33 \leq \beta \leq \frac{7}{9}$. Further the coefficient of $m_{i}$ is always nonpositive for $0.23 \leq \beta \leq \frac{7}{9}$. Thus the excess of the positive value of coefficient of $m_{i}$ over the sum of the positive values of the coefficients of $m_{i-1}$ and $m_{i+1}$ is $L_{5}(\beta)=\frac{\left(-2+11 \beta-9 \beta^{2}\right)}{2}$, for $0.23 \leq \beta \leq 0.33$, $L_{6}(\beta)=-1+6 \beta-6 \beta^{2}$, for $0.33 \leq \beta \leq 0.67$ and $L_{7}(\beta)=\frac{\beta(7-9 \beta)}{2}$, for $0.67 \leq \beta \leq \frac{7}{9}$.

It may be seen easily that $L_{1}(\beta), L_{2}(\beta), L_{3}(\beta), L_{4}(\beta)$, $L_{5}(\beta), L_{6}(\beta)$ and $L_{7}(\beta)$ are positive for corresponding values of $\beta$. Thus, the coefficient matrix A of the system of equations (6.2.13) is invertible and its rowmax norm for $\alpha=\frac{1}{3}$ (or $\frac{2}{3}$ or $\frac{1}{2}$ ), that is,

$$
\mathrm{P} A^{-1} \mathrm{P} \leq K_{2}
$$

Where

$$
\begin{gathered}
K_{2}=\max _{\beta}\left\{L_{1}^{-1}(\beta), L_{2}^{-1}(\beta), L_{3}^{-1}(\beta), L_{4}^{-1}(\beta),\right. \\
\left.L_{5}^{-1}(\beta), L_{6}^{-1}(\beta), L_{7}^{-1}(\beta)\right\}
\end{gathered}
$$$$
\text { Let } K^{*}=\max \left\{K_{1}, K_{2}\right\}
$$

\section{DISCRETE ERROR BOUNDS}

For a given $h>0$, we introduce the set,

$$
R_{h}=\left\{x_{0}+j h: j \text { is an integer }\right\}
$$

and define a discrete interval $[0,1]_{h}=[0,1] \bigcap R_{h}$.

$$
\omega_{h}\left(D_{h}{ }^{1} f, p\right) \text { for the modulus of continuity of } \mathrm{f}
$$

defined over $[0,1]_{h}$, whereas the discrete norm of a function f over the interval $[0,1]_{h}$

$$
\mathrm{P} f \mathrm{P}^{*}=\max _{[0,1]}|f(x)| \text {. }
$$

For a function $\mathrm{f}$ and distinct points $x_{1}, x_{2}, x_{3}$ in its domain the first and second divided differences are defined as

$$
\left[x_{1}, x_{2}\right] f=\frac{\left\{f\left(x_{1}\right)-f\left(x_{2}\right)\right\}}{x_{1}-x_{2}}
$$

and

$$
\left[x_{1}, x_{2}, x_{3}\right] f=\frac{\left[x_{1}, x_{2}\right] f-\left[x_{2}, x_{3}\right] f}{\left(x_{1}-x_{3}\right)}
$$

respectively. Similarly, higher order divided differences can be defined.

For convenience we write $f^{(1)}$ for $D_{h}^{(1)} f, f_{i}^{(1)}$ for $D_{h}{ }^{(1)} f\left(x_{i}\right)$, Without assuming any smoothness condition on the data $\mathrm{f}$, obtaining the following bounds for the error function $e^{(k)}(x)=D_{h}^{(k)} s(x, h)-f^{(k)}(x)$ over the discrete interval $[0,1]_{h}$.

In order to show convergence of a discrete spline, the following lemma is required, given by Lyche[7]

Lemma 3.1: Let $\left\{a_{j}\right\}_{j=1}^{m}$ and $\left\{b_{j}\right\}_{j=1}^{n}$ be given sequences of nonnegative real numbers such that $\sum_{i=1}^{m} a_{i}=\sum_{j=1}^{n} b_{j}$, then for any real valued function $\mathrm{f}$, defined on a discrete interval $[0,1]_{h}$, we have

$$
\begin{gathered}
\left|\sum_{i=1}^{m} a_{i}\left[x_{i 0}, x_{i 1}, \ldots, x_{i k}\right]\right| f-\left|\sum_{j=1}^{n} b_{j}\left[y_{j 0}, y_{j 1}, \ldots, y_{j k}\right]\right| f \\
\leq \omega_{h}\left(D_{h}^{(k)} f,|1-k h|\right) \sum \frac{a_{i}}{k !}
\end{gathered}
$$

where $x_{i k}, y_{j k} \in[0,1]_{h}$, for relevant values of $\mathrm{i}, \mathrm{j}$ and $\mathrm{k}$.

Theorem 3.2: Suppose $\mathrm{s}(\mathrm{x}, \mathrm{h})$ is the deficient discrete cubic spline interpolant of theorem 2.1 then,

$$
\mathrm{P}\left(e_{i}^{(1)}\right) \mathrm{P} \leq\left(\frac{1}{2 !}\right) K(\alpha, \beta, p, h) \omega_{h}\left(f^{(2)},|1-2 p|\right)
$$

proof: Writing $m_{i}-f_{i}^{(1)}=e_{i}^{(1)}$, from $(2.13)$

$$
\begin{aligned}
& {\left[\frac{2(1-\alpha)(1-\beta) h^{2}}{p}+R_{i} p\right]\left(m_{i-1}-f_{i-1}{ }^{(1)}\right)} \\
& +\left[\frac{2 \beta(1-\alpha) h^{2}}{p}+\frac{2 \alpha(1-\beta) h^{2}}{p}\right. \\
& \left.-\left(R_{i}+R_{i}^{*}-1+6 \beta-6 \beta^{2}\right) p\right]\left(m_{i}-f_{i}^{(1)}\right) \\
& +\left[\frac{2 \alpha \beta h^{2}}{p}+R_{i}^{*} p\right]\left(m_{i+1}-f_{i+1}{ }^{(1)}\right)
\end{aligned}
$$




$$
\begin{aligned}
= & F_{i}-\left[\frac{2(1-\alpha)(1-\beta) h^{2}}{p}+R_{i} p\right] f_{i-1}{ }^{(1)} \\
& -\left[\frac{2 \beta(1-\alpha) h^{2}}{p}+\frac{2 \alpha(1-\beta) h^{2}}{p}\right. \\
& \left.-\left(R_{i}+R_{i}^{*}-1+6 \beta-6 \beta^{2}\right) p\right] f_{i}^{(1)} \\
& -\left[\frac{2 \alpha \beta h^{2}}{p}+R_{i}^{*} p\right] f_{i+1}^{(1)}
\end{aligned}
$$

or (3.2) can be written as following

$$
A\left(e_{i}^{(1)}\right)=F_{i}-A\left(f_{i}^{(1)}\right)=D_{i}
$$

where $D_{i}(f)$ or $D_{i}$ is

$$
\begin{aligned}
D_{i}= & -6 \beta(1-\beta) p \frac{\left(f\left(\alpha_{i+1}\right)-f\left(\alpha_{i}\right)\right)}{p}+\left\{\alpha^{2}(3-2 \alpha) p\right. \\
& \left.+\frac{2 \alpha h^{2}}{p}\right\} D_{h}^{(1)} f\left(\beta_{i+1}\right)+\left\{\frac{2(1-\alpha) h^{2}}{p}+p\right. \\
& \left.-\alpha^{2}(3-2 \alpha) p\right\} D_{h}^{(1)} f\left(\beta_{i}\right)-\left\{\frac{2(1-\alpha)(1-\beta) h^{2}}{p}\right. \\
& \left.+R_{i} p\right\} D_{h}^{(1)} f\left(x_{i-1}\right)+\left\{\left(R_{i}+R_{i}^{*}-1+6 \beta-6 \beta^{2}\right) p\right. \\
& \left.-\frac{2 \beta(1-\alpha) h^{2}}{p}-\frac{2 \alpha(1-\beta) h^{2}}{p}\right\} D_{h}^{(1)} f\left(x_{i}\right) \\
& -\left\{\frac{2 \alpha \beta h^{2}}{p}+\alpha^{2} \beta(3 \beta-2 \alpha) p\right\} D_{h}^{(1)} f\left(x_{i+1}\right)
\end{aligned}
$$

A little computation shows that $D_{i}$ may be rewritten in the form of divided difference as following

$$
\left|D_{i}(f)\right|=\left|\sum_{i=1}^{11} u_{i}\left[x_{i 0}, x_{i 1}, x_{i 2}\right]\right| f-\left|\sum_{j=1}^{11} v_{j}\left[y_{j 0}, y_{j 1}, y_{j 2}\right]\right| f
$$

with

$$
\begin{aligned}
& u_{1}=6 \beta p(p+h), \quad u_{2}=6 \beta^{2} p(h+\alpha p), \\
& u_{3}=6 \alpha \beta^{2} p^{2} \quad u_{4}=u_{5}=\alpha^{2}(3-2 \alpha) p^{2}, \\
& u_{6}=u_{7}=v_{4}=v_{5}=2 \beta(1-\alpha) h^{2} \\
& u_{8}=u_{9}=v_{6}=v_{7}=2 \alpha \beta h^{2}, \\
& u_{10}=u_{11}=R_{i} p^{2} \quad v_{1}=6 \beta p(h+\alpha p),
\end{aligned}
$$

$$
\begin{aligned}
& v_{2}=6 \alpha \beta p^{2} \quad v_{3}=6 \beta^{2} p(p+h), \\
& v_{8}=v_{9}=p^{2}(1-\beta) \quad v_{10}=v_{11}=R_{i}^{*} p^{2}, \\
& x_{10}=x_{i}+h \quad x_{11}=x_{20}=y_{12}=y_{21}=\alpha_{i+1} \text {, } \\
& x_{12}=x_{21}=x_{30}=y_{22}=y_{30}=\alpha_{i} \text {, } \\
& x_{22}=x_{31}=x_{82}=x_{91}=x_{100}=y_{10}=y_{31} \\
& =y_{42}=y_{51}=y_{60}=y_{82}=y_{91}=y_{100}=x_{i}-h \text { ' } \\
& x_{32}=x_{92}=x_{101}=x_{110}=y_{11}=y_{20}=y_{32}=y_{52} \\
& =y_{61}=y_{70}=y_{92}=y_{101}=y_{110}=x_{i}+h
\end{aligned}
$$$$
x_{40}=x_{80}=\beta_{i+1}-h \text {, }
$$$$
x_{41}=x_{50}=x_{81}=x_{90}=\beta_{i+1}+h \text {, }
$$$$
x_{42}=x_{51}=x_{61}=x_{70}=y_{80}=\beta_{i}-h \text {, }
$$$$
x_{52}=x_{60}=y_{81}=y_{90}=\beta_{i}+h \text {, }
$$$$
x_{62}=x_{71}=y_{41}=x_{i-1}+h \text {, }
$$$$
x_{72}=x_{102}=x_{111}=y_{40}=y_{50}=x_{i-1}-h,
$$$$
x_{112}=y_{72}=y_{112}=x_{i+1}+h \text {, }
$$$$
y_{62}=y_{71}=y_{102}=y_{111}=x_{i+1}-h \text {. }
$$

Observing the fact that $\sum u_{i}=\sum v_{j}=N^{*}(\alpha, \beta, p, h)$ applying Lemma 3.1 in (3.4) for $m=11$ and $n=11$ and $k=2$ to see that

$$
\left|D_{i}\right| \leq\left(\frac{1}{2 !}\right) N^{*}(\alpha, \beta, p, h) \omega_{h}\left(f^{(2)},|1-2 p|\right)
$$

Now using the equations (2.16) and (3.6) in (3.3),

$$
\mathrm{P} e^{(1)}(x) \mathrm{P} \leq\left(\frac{1}{2 !}\right) K(\alpha, \beta, p, h) \omega_{h}\left(f^{(2)},|1-2 p|\right)
$$

where $K(\alpha, \beta, p, h)=K^{*} N^{*}(\alpha, \beta, p, h)$ is some positive functions of $\alpha, \beta, p, h$. 


\section{CONCLUSION}

In this paper it is proved that there exist a deficient discrete cubic spline interpolant by matching the given function and its first order difference at the intermediate points between successive mesh points for a uniform mesh and studied its error bounds. This concept can be applied for higher dimensional splines as a future work.

\section{ACKNOWLEDGMENTS}

The authors would like to thanks referees of this paper for their valuable suggestions.

\section{REFERENCES}

[1] Astore, P. H. and Duries, C.S. 1974 Discrete splines, Numer. Math., 22, 393-402.

[2] Boneva, K., Kendall, W. and Stefanov, I. 1971 Spline transformation, j. Roy. Statist. Soc. Ser. B. 33, $1-70$

[3] Dikshit, H.P. and Powar, P.L. 1982 Discrete cubic spline interpolation, Numer, Math.40, 71-78.

[4] Dikshit, H.P. and Rana, S.S. 1987 Discrete cubic spline interpolation over a non-uniform mesh, Rocky Mountain Jour. of Math, 17, $709-718$.
[5] Dikshit, H.P., Sharma, A. and Tzimbalario, J. 1984 Asymptotic error expansion for spline interpolation,Canada. Math. Bull. 27, 337-344.

[6] Jia , R. Q. 1983 Total positivity of the discrete spline collocation matrix, J. Approx. Theo.39, 15- 23.

[7] Lyche, T. 1975 Discrete cubic spline interpolation, Report RRI 5, University of Oslo.

[8] Lyche, T. 1976 Discrete cubic spline interpolation, BIT, $16,281-290$.

[9] Malcom, M. A. 1973 Nonlinear spline functions, lquad Report Stam. CS, Stanford Univ. 197, 372-373.

[10] Mangasarian, O.L. and Schumaker, L.L. 1971 Discrete spline via mathematical programming, SIAM Jour. Control , 9, $174-183$.

[11] Rana, S.S. 1984 Discrete cubic X-spline interpolation,J. Orissa Math. Soc., 3, $67-75$.

[12] Rana, S.S., and Dubey, Y.P.,Local behaviour of the deficient discrete cubic spline interpolation, Jour. Approx. Theo., 86(1996), 120 - 127.

[13] Schumaker, L. L. 1973 Constructive aspects of discrete polynomial spline functions, J. of Approx. Theory (G.G.Lorcut 2 ed.), Academic Press, New York. 